\title{
Critical Point Inequalities and Scaling Limits
}

\author{
Charles M. Newman* \\ Department of Mathematics, Indiana University, Bloomington, Indiana 47405, USA
}

\begin{abstract}
A refined and extended version of the Buckingham-Gunton inequality relating various pairs of critical exponents is shown to be valid for a large class of statistical mechanical models. If this inequality is an equality (in the refined sense) and one of the critical exponents has a non-Gaussian value, then any scaling limit must be non-Gaussian. This result clarifies the relationship between the nontriviality or triviality of the scaling limit for ordinary critical points in four dimensions (or tricritical points in three dimensions) and the existence of logarithmic factors in the asymptotics which define the two critical exponents.
\end{abstract}

\section{General Results}

In this paper, we use some surprisingly simple probabilistic arguments to obtain rather detailed information about the critical behavior of a fairly large class of statistical mechanical systems. For example, in the case of a four dimensional Ising model we relate (see Corollary 2.7 below) the possible existence of logarithmic factors in the large distance behavior of the two point correlation (at the critical point) and in the small external field behavior of the free energy (at the critical temperature) to the question of the possible triviality (i.e., Gaussian-ness) of the Kadanoff block spin scaling limit $[14,22,21]$. One view of this result is that it provides sufficient conditions for proving that the scaling limit is nontrivial and thus perhaps also for proving that nontrivial $\phi^{4}$ field theories exist in four dimensional spacetime $[2,15,31]$. This point of view underlies many of the critical exponent results of Glimm and Jaffe (see [16] and the references listed there). Another view is that it merely yields a new critical exponent inequality relating the two possible logarithmic factors - an inequality which must be strict if the scaling limit is to be Gaussian (as it "should" be). This latter view is consistent with various calculations which imply such a strict inequality (see the discussion following Corollary 2.8 below and the references cited there).

* Alfred P. Sloan Research Fellow. Research supported in part by National Science Foundation Grant MCS 77-20683 
In this section, we first present our results (without proofs) in the rather general probabilistic context of a sequence of random variables, $S_{n}$ (and an associated sequence of positive numbers, $V_{n}$ ); the proofs are given in Sect. III. In Sect. II, we discuss in some detail various statistical mechanical models to which our general results apply and present these applications using standard critical point terminology and notation. One type of application [see (2.24) and Corollaries 2.6-2.8] concerns the Buckingham-Gunton inequality $[6,9]$ relating the magnetic susceptibility exponent $\delta$ and the spin-spin correlation exponent $\eta$. Another application (see Corollaries 2.9-2.10) yields an inequality relating the specific heat exponents $\alpha, \alpha^{\prime}$ and the energy-energy correlation exponent $\eta_{E}: 2-\eta_{E} \leqq d \bar{\alpha} /(2-\bar{\alpha})$ where $\bar{\alpha}=\max \left(\alpha, \alpha^{\prime}\right)$; this improves (at least when $\alpha^{\prime} \geqq \alpha$ ) Fisher's inequality, $2-\eta_{E} \leqq d \alpha^{\prime}$ [9]. A third application (see the remark following Proposition 2.11) yields in $\lambda P(\phi)$ field theory models an inequality relating the critical exponent of the vacuum energy density $\alpha_{\infty}(\lambda)$ as $\lambda \rightarrow \lambda_{c}$ to the $P(\phi)-P(\phi)$ correlation exponent. We demonstrate in Sect. II that our general results are applicable to systems satisfying either the FKG inequalities (Proposition 2.5) or the GKS inequalities (see the discussion preceeding Corollary 2.9) or reflection positivity (Proposition 2.11). These general results and their proofs are so simple that we feel the techniques may very well be useful in treating other models not discussed in this paper (see [25] and the discussion at the end of Sect. II).

In the general presentation of this section of the paper, we point out that $S_{n}$ typically represents the (random) amount of some extensive quantity (such as magnetization, energy, or number of particles) contained in a finite region $\Lambda_{n} \subset \mathbb{R}^{d}$ of volume $V_{n}$. We denote by $z$ the intensive thermodynamic variable (such as external magnetic field, inverse temperature, or (the negative of the) chemical potential) associated with the extensive quantity $S_{n}$.

We next list several hypotheses concerning $S_{n}, V_{n}$, and

$$
\sigma_{n}=\left(\operatorname{var}\left(S_{n}\right)\right)^{1 / 2}=\left[E\left(S_{n}^{2}\right)-\left(E\left(S_{n}\right)\right)^{2}\right]^{1 / 2} \text {, }
$$

which will be assumed at various points throughout the paper. A discussion of the nature of these hypotheses follows Corollary 2.4 below.

Hypothesis $A: \exists \varepsilon>0$ so that

$$
f_{n}(z) \equiv\left(V_{n}\right)^{-1} \log E\left(\exp \left(z S_{n}\right)\right) \rightarrow f(z), \quad z \in(-\varepsilon, \varepsilon) ;
$$

$f(z)$ is necessarily a real convex function on $(-\varepsilon, \varepsilon)$ with $f(0)=0$.

$$
\begin{aligned}
& \text { Hypothesis } B: f^{\prime}(0) \text { exists. } \\
& \text { Hypothesis } C: \quad E\left(S_{n}\right) / V_{n} \text { is independent of } n . \\
& \text { Hypothesis } D: V_{n} \rightarrow \infty . \\
& \text { Hypothesis } E: \quad \sigma_{n}>0 \text { for all } n . \\
& \text { Hypothesis } F: \quad \sigma_{n} \rightarrow \infty . \\
& \text { Hypothesis } G: \sigma_{n} \rightarrow \infty \text { and } \limsup _{n} \sigma_{n+1} / \sigma_{n}<\infty . \\
& \text { Hypothesis } H: f_{e}(z) \equiv f(z)+f(-z) \text { satisfies } \\
& \sup _{z>0}\left(\limsup _{t \rightarrow 0} f_{e}(t z) /\left(z^{p} f_{e}(t)\right)\right)<\infty ;
\end{aligned}
$$

here $p$ is some real number.

Hypothesis I: $f_{n}(z) \leqq f(z) \forall n, \quad z \in(-\varepsilon, \varepsilon)$. 
We write $X_{n} \stackrel{w}{\longrightarrow} X$ (weak convergence or convergence in distribution) if $E\left(g\left(X_{n}\right)\right) \rightarrow E(g(X))$ for every bounded continuous function $g$, or equivalently if $P\left(X_{n} \leqq x\right) \rightarrow P(X \leqq x)$ for every $x$ such that $P(X=x)=0$. We define (for $\sigma_{n}>0$ )

$$
X_{n}=\left(S_{n}-E\left(S_{n}\right)\right) / \sigma_{n}
$$

and note that since $\sup _{n} E\left(X_{n}^{2}\right)<\infty$, it follows that $\left\{X_{n}\right\}$ is weakly sequentially compact; i.e., every subsequence of $\left\{X_{n}\right\}$ has a weakly convergent subsubsequence. We also define (when Hypothesis $B$ is valid)

$$
\begin{aligned}
& f_{+}(z)=f(z)-f^{\prime}(0) z \\
& f_{-}(z)=f(-z)+f^{\prime}(0) z=f_{+}(-z) .
\end{aligned}
$$

Note that $f_{e}=f_{+}+f_{-}$and that $f_{+}$and $f_{-}$are non-negative nondecreasing functions on $[0, \infty)$ with $f_{+}(0)=f_{-}(0)=0$.

Theorem 1.1. Assume Hypotheses $A, B$, and $D$. Then

$$
S_{n} / V_{n} \stackrel{w}{\longrightarrow} f^{\prime}(0) \text {. }
$$

Theorem 1.2. Assume Hypotheses $A, B, C, E$, and I. Then

$$
\liminf _{n \rightarrow \infty} V_{n} f_{e}\left(z / \sigma_{n}\right)>0 \text { for any } z \neq 0 \text {. }
$$

Theorem 1.3. Assume Hypotheses A, B, C, E, and I and assume also that

$$
\limsup _{n \rightarrow \infty} V_{n} f_{e}\left(z / \sigma_{n}\right)<\infty, \text { for some } z \neq 0 .
$$

Then

$$
\liminf _{n \rightarrow \infty} V_{n} f_{ \pm}\left(z / \sigma_{n}\right)>0, \text { for any } z \neq 0 .
$$

If in addition to the above assumptions, we assume Hypothesis $G$, then

$$
0<\liminf _{z \rightarrow 0} \frac{f_{+}(z)}{f_{-}(z)} \leqq \limsup _{z \rightarrow 0} \frac{f_{+}(z)}{f_{-}(z)}<\infty .
$$

Theorem 1.4. Assume Hypotheses $A, B, C, F, H$, and $I$ and assume also that (1.8) is valid. Then $p \in[1,2]$ (where $p$ is the number appearing in Hypothesis $H$ ) and any limit in distribution $X$ of $\left\{X_{n} \equiv\left(S_{n}-E\left(S_{n}\right)\right) / \sigma_{n}\right\}$ satisfies for some $c>0$ :

$$
\begin{aligned}
& E(X)=0, \quad E\left(X^{2}\right)=1 ; \\
& \left.\begin{array}{l}
E(\exp (t X)) \leqq \exp \left(|c t|^{p} / p\right), \quad t \in(-\infty, \infty) ; \\
P(X \geqq x) \\
P(X \leqq-x)
\end{array}\right\} \leqq \exp \left(-(x / c)^{q} / q\right), \quad x \geqq 0,
\end{aligned}
$$

where $q \in[2, \infty]$ satisfies $(1 / q)+(1 / p)=1$ (and for the case $p=1, q=\infty,(1.13)$ is to be interpreted as $P(|X|>c)=0)$. Thus, if $p \neq 2, X$ is non-Gaussian. The conclusions of the theorem remain valid if instead of Hypothesis $H$ and (1.8) we assume the existence of $\bar{f}(z)$ such that $f_{e}(z) \leqq \bar{f}(z)$ for small $|z|$ and such that (1.3) and (1.8) are valid with $f_{e}$ replaced by $\bar{f}$. 


\section{Applications to Various Models}

In this section, we consider a simple $d$-dimensional cubic lattice of random variables $\left\{Y_{i}: i=\left(i_{1}, \ldots, i_{d}\right) \in \mathbb{Z}^{d}\right\}$ and the corresponding "block" variables

$$
S_{n}=\sum_{i_{1}, \ldots, i_{d}=1}^{n} Y_{i}
$$

Our results could be applied to rather general lattices and blocks, but we prefer to restrict attention to the simplest interesting case. In this context, we take $V_{n}=n^{d}$ and note that

$$
\sigma_{n}^{2}=\sum_{i_{1}, \ldots, i_{d}=1}^{n} \sum_{j_{1}, \ldots, j_{d}=1}^{n}\left(E\left(Y_{i} Y_{j}\right)-E\left(Y_{i}\right) E\left(Y_{j}\right)\right) .
$$

We will henceforth automatically assume Hypotheses A, B, C, F, and I. We note that Hypothesis $\mathrm{C}$ is implied by the assumption that $E\left(Y_{i}\right)$ is independent of $i$. When the summand in (2.2) depends only on $i-j$ we may define the functions $F$ on $\mathbb{Z}^{d}$ and $G$ on $[0, \infty)$ by

$$
\begin{aligned}
& \operatorname{cov}\left(Y_{i}, Y_{j}\right) \equiv E\left(Y_{i} Y_{j}\right)-E\left(Y_{i}\right) E\left(Y_{j}\right)=F(i-j), \\
& G(r)=\sum_{|i| \leqq R} F(i),
\end{aligned}
$$

where $|\cdot|$ denotes Euclidean length in $\mathbb{R}^{d}$. We also note that if full translation invariance (of the joint distributions) of $\left\{X_{i}\right\}$ is assumed, then the "block", $\{i: 1$ $\left.\leqq i_{1}, \ldots, i_{d} \leqq n\right\}$, can be translated to a block $\Lambda_{n}$ centered near the origin so that $\Lambda_{n} \rightarrow \mathbb{Z}^{d}$ and $f(z)$ represents a (full) infinite volume thermodynamic quantity. In many applications $F(i) \geqq 0 \forall i$ and then the asymptotics of $\sigma_{n}$ are closely related to those of $G(R)$ as seen in the following easily proven proposition:

Proposition 2.1. Suppose (2.3) is valid and $F(i) \geqq 0 \forall i$ (and $F(0)>0$ ); then $\exists c_{1}, c_{2}$, $K_{1}, K_{2} \in(0, \infty)$ such that for all $n$,

$$
K_{1} n^{d} G\left(c_{1} n\right) \leqq \sigma_{n}^{2} \leqq K_{2} n^{d} G\left(c_{2} n\right) .
$$

If in addition, $G$ satisfies

$$
\limsup _{t \rightarrow \infty} G(t R) / G(t)<\infty, \text { some } R>1 ;
$$

then Hypothesis $\mathrm{G}$ is valid.

In order to relate (1.7)-(1.8) to critical exponent inequalities, we state several corollaries of our theorems in which we assume various versions of the asymptotics: $f(z) \sim|z|^{p}$, small $z ; \sigma_{n} \sim n^{(d+2-\eta) / 2}$, large $n$ (which, if Proposition 2.1 applies, is rigorously equivalent to $G(R) \sim R^{2-\eta}$ and heuristically equivalent to $F(i)$ $\left.\sim|i|^{-(d-2+\eta)}\right)$.

Corollary 2.2. Suppose we define $p$ and $\eta b y$

$$
\begin{aligned}
& p=\liminf _{z \rightarrow 0+} \log f_{e}(z) / \log z \\
& (d+2-\eta) / 2=\limsup _{n \rightarrow \infty} \log \sigma_{n} / \log n ;
\end{aligned}
$$


then $p \geqq 1$ and

$$
2-\eta \leqq \frac{2-p}{p} d
$$

Remark. As we explain below [see (2.24)], (2.9) in the appropriate context is just the Buckingham-Gunton inequality $[6,9]$.

Proof. If $p<1$ then there is a sequence $z_{n} \rightarrow 0+$, such that for some $\varepsilon>0$, $\log f_{e}\left(z_{n}\right) / \log z_{n}<1-\varepsilon$ and therefore $f_{e}\left(z_{n}\right)>\left(z_{n}\right)^{1-\varepsilon}$ for large $n$. But this contradicts the fact that $f_{e}\left(z_{n}\right) / z_{n} \rightarrow 0$ [since $\left.f_{e}^{\prime}(0)=0\right]$; thus $p \geqq 1$. To obtain (2.9), we note that (2.7)-(2.8) imply that

$$
\begin{aligned}
& f_{e}(z)<|z|^{p^{\prime}}, \text { any } p^{\prime}<p, \text { small } z, \\
& \sigma_{n_{k}}>\left(n_{k}\right)^{r}, \text { any } r<(d+2-\eta) / 2, \text { for some subsequence } n_{k} .
\end{aligned}
$$

Thus by (1.7) with $V_{n}=n^{d}$, we have that $d-p^{\prime} r \geqq 0$ and so $d-p(d+2-\eta) / 2 \geqq 0$ which is equivalent to (2.9).

Corollary 2.3. Let $N \in\{1,2,3, \ldots\}$ be fixed and suppose the $p$ and $\eta$ defined in Corollary 2.2 satisfy the equality, $2-\eta=\frac{2-p}{p} d$. Suppose in addition that there are real valued constants, $\theta_{1}, \ldots, \theta_{N}, \psi_{1}, \ldots, \psi_{N}$, such that for some $K, \varepsilon \in(0, \infty)$

$$
\begin{aligned}
& f_{e}(z) \leqq K|z|^{p}|\log | z||^{\theta_{1}} \cdot\left|\log _{2}\right| z||^{\theta_{2}} \ldots\left|\log _{N}\right| z||^{\theta_{N}}, \text { small } z, \\
& \sigma_{n}^{2} \geqq \varepsilon n^{(d+2-\eta)}(\log n)^{\psi_{1}} \cdot\left(\log _{2} n\right)^{\psi_{2}} \ldots\left(\log _{N} n\right)^{\psi_{N}}, \text { large } n
\end{aligned}
$$

where $\log _{2} n=\log (\log n), \log _{k+1} n=\log \left(\log _{k} n\right)$. If $\psi_{j}=2 \theta_{j} / p\left(=(d+2-\eta) \theta_{j} / d\right)$ for $j=1, \ldots, N-1$, then

$$
\psi_{N} \leqq 2 \theta_{N} / p \text {. }
$$

Proof. This corollary is a straightforward consequence of (1.7).

Corollary 2.4. Let $N \in\{1,2,3, \ldots\}$ be fixed. Suppose for some $p, \theta_{1}, \ldots, \theta_{N}$, and $K<\infty$,

$$
f_{e}(z) \leqq K|z|^{p}|\log | z\left\|^{\theta_{1}} \ldots\right\| \log _{N} \mid z \|^{\theta_{N}}, \quad \text { small } z .
$$

Then for some $K^{\prime}<\infty$

$$
\sigma_{n}^{2} \leqq K^{\prime} n^{2 d / p}(\log n)^{2 \theta_{1} / p} \ldots\left(\log _{N} n\right)^{2 \theta_{N} / p}, \text { large } n ;
$$

if for some $\varepsilon>0$,

$$
\sigma_{n}^{2} \geqq \varepsilon n^{2 d / p}(\log n)^{2 \theta_{1} / p} \ldots\left(\log _{N} n\right)^{2 \theta_{N} / p}, \quad \text { large } n,
$$

then $p \in[1,2]$ and any limit in distribution $X$ of $\left\{\left(S_{n}-E\left(S_{n}\right)\right) / \sigma_{n}\right\}$ satisfies $(1.11)-(1.13)$ for some $c>0 . X$ is non-Gaussian if $p \neq 2$.

Proof. This corollary follows directly from (1.7) and Theorem 1.4. 
We now proceed to apply the above theorems and corollaries to various statistical mechanical models, $\left\{Y_{i}: i \in \mathbb{Z}^{d}\right\}$. Hypotheses A-F are ones which either are or ought to be valid at any respectable critical point [except that Hypothesis $B$ should fail if the pure critical phase in question can coexist with some other (critical or non-critical) pure phase]. Hypotheses $\mathrm{G}-\mathrm{H}$ (which are not needed for many of our applications) are perhaps not quite as reasonable as the previous ones but are still fairly natural assumptions about the nature of $f(z)$ and $\sigma_{n}$ [or $\left.G(R)\right]$ at a critical point. Hypothesis I (which is essential for our results) stands out as the only "technical" assumption of the lot and we view our results as applicable to any reasonable (critical) system for which Hypothesis I is valid. We proceed to discuss several such systems : Ising models, lattice gases, and Euclidean field theories. We shall see that Hypothesis I can be derived from various correlation inequalities or from reflection positivity; it is thus in principle not restricted to any narrow class of models (such as purely ferromagnetic ones) and may perhaps be valid even for continuum models of fluids. The list of models we present here is not meant to be exhaustive.

We first consider Ising models (and the related lattice gas models) in which the FKG inequalities are valid. Suppose $\left\{Y_{i}\right\}$ is obtained as a limit (in the sense of weak convergence of finite dimensional distributions) of finite systems $\left\{Y_{1}^{m}, \ldots, Y_{m}^{m}\right\}$ whose joint distribution on $\mathbb{R}^{m}$ is proportional to

$$
\exp \left(\sum_{k=1}^{m} h_{k} y_{k}+\sum_{j, k=1}^{m} J_{j k} y_{j} y_{k}\right) \prod_{k=1}^{m} d \varrho_{k}\left(y_{k}\right),
$$

where $J_{j k} \geqq 0, h_{k} \in \mathbb{R}$, and $\varrho_{k}$ are arbitrary measures on $\mathbb{R}^{1}$; then $\left\{Y_{i}\right\}$ will satisfy the FKG inequalities $[12,32]$ as defined in the next proposition. We say that a real valued function $g$ on $\mathbb{R}^{m}$ is increasing if $g\left(y_{1}, \ldots, y_{m}\right) \leqq g\left(x_{1}, \ldots, x_{m}\right)$ whenever $y_{j} \leqq x_{j}$ for $j=1, \ldots, m$.

Proposition 2.5. Assume $\left\{Y_{i}: i \in \mathbb{Z}^{d}\right\}$ satisfies the $F K G$ inequalities; i.e., for any $m$, any $i_{1}, \ldots, i_{m} \in \mathbb{Z}^{d}$, and any increasing functions $g_{1}, g_{2}$ on $\mathbb{R}^{m}$ (such that $\left.\left.E\left[\left(g_{j}\left(Y_{i_{1}}, \ldots, Y_{i_{m}}\right)\right)^{2}\right]<\infty ; j=1,2\right)\right)$,

$$
\operatorname{cov}\left(g_{1}\left(Y_{i_{1}}, \ldots, Y_{i_{m}}\right), \quad g_{2}\left(Y_{i_{1}}, \ldots, Y_{i_{m}}\right)\right) \geqq 0 .
$$

Assume further that $\left\{Y_{i}: i \in \mathbb{Z}^{d}\right\}$ is translation invariant in the sense that for any $j \in \mathbb{Z}^{d}$ $\left\{Y_{i}^{\prime} \equiv Y_{i+j}: i \in \mathbb{Z}^{d}\right\}$ is equidistributed with $\left\{Y_{i}: i \in \mathbb{Z}^{d}\right\}$. Then if Hypothesis $A$ is valid, so is Hypothesis I.

Proof. For $k=1, \ldots, d$, we define $\Theta_{k}: \mathbb{Z}^{d} \rightarrow \mathbb{Z}^{d}$ to be reflection about the plane $i_{k}=1 / 2$ :

$$
\left(\Theta_{k} i\right)_{l}=\left\{\begin{array}{ccc}
i_{l}, & \text { if } l \neq k \\
-i_{l}+1, & \text { if } l=k
\end{array}\right.
$$

and we define for $I$ a finite subset of $\mathbb{Z}^{d}$ and $T=\sum_{i \in I} Y_{i}, \Theta_{k} T=\sum_{i \in I} Y_{\Theta_{k} i}$. For any such $T$, (2.19) for $g_{1}= \pm \exp ( \pm|z| T), g_{2}= \pm \exp \left( \pm|z| \Theta_{k} T\right)$ implies

$$
E\left(e^{z\left(\Theta_{k} T+T\right)}\right) \geqq E\left(e^{z \Theta_{k} T}\right) E\left(e^{z T}\right), \quad z \in \mathbb{R} .
$$


We define iteratively $T_{0}=S_{n}, T_{k}=\Theta_{k} T_{k-1}+T_{k-1}$ and use (2.21) $d$ times together with the fact that because of translation invariance,

$$
\begin{aligned}
& E\left(\exp \left(z \Theta_{k} T_{k-1}\right)\right)=E\left(\exp \left(z T_{k-1}\right)\right) \text { for each } k, \text { to obtain } \\
& E\left(\exp z T_{d}\right) \geqq\left[E\left(\exp \left(z T_{0}\right)\right)\right]^{2^{d}}, \quad z \in \mathbb{R} .
\end{aligned}
$$

Now $T_{0}=S_{n}$ while by translation invariance, $E\left(\exp \left(z T_{d}\right)\right)=E\left(\exp \left(z S_{2 n}\right)\right)$. Thus taking the logarithm of (2.22) and dividing by $(2 n)^{d}$ yields

$$
f_{2 n}(z) \geqq f_{n}(z), \quad z \in \mathbb{R},
$$

which, by Hypothesis A, implies Hypothesis I.

In the Ising model context, the critical exponent $p$ (of Corollaries 2.2-2.4) is usually expressed as $p=1+1 / \delta$ with $\delta \in[1, \infty)$ [for $p \in(1,2)]$. Inequality (2.9) then becomes the usual Buckingham-Gunton Inequality $[6,9]$ :

$$
2-\eta \leqq \frac{\delta-1}{\delta+1} d .
$$

The classical (mean-field) value of $\delta$ is 3 for an ordinary critical point (or $2 k-1$ for a "type- $k$ " critical point) (see, e.g., $[4,7,8]$ ). The classical (Ornstein-Zernike) value of $\eta$ is $\eta=0$ (for any $k$ ). For short range Ising models in $d$-dimensions, it is believed $[10,35,36]$ that there is a critical dimension $d_{c}$ [with $d_{c}=4$ for ordinary critical points, $d_{c}=2 k /(k-1)$ for type- $k$ critical points] so that for $d>d_{c}, \delta$ and $\eta$ take on their classical values [so that (2.24) becomes a strict inequality] and the Kadanoff scaling limit is Gaussian [so that any limit $X$ of $\left(S_{n}-E\left(S_{n}\right)\right) / \sigma_{n}$ is Gaussian]. For $d$ $<d_{c}$, it is thought (with some dissent [3]) that $\delta$ and $\eta$ do not have their classical values, that (2.24) is an equality (with no logarithmic terms, such as those given in Corollaries 2.3-2.4, needed to describe the asymptotics of $f_{e}$ as $z \rightarrow 0$ or $\sigma_{n}$ as $n \rightarrow \infty$ ), and that the scaling limit is non-Gaussian. The next corollary gives sufficient conditions for proving rigorously that the scaling limit is non-Gaussian; according to the above discussion, it should be applicable to ordinary critical points in 2 and 3 dimensions, and to arbitrary type critical points in 2 dimensions. We discuss the case $d=d_{c}$ following this corollary. Recall that we automatically assume Hypotheses A, B, C, F, and I. We also replace $z$ by $h$ since this variable represents external magnetic field in the Ising model context.

Corollary 2.6. Assume the validity of (2.5) [for some $\left.c_{1}, c_{2}, K_{1}, K_{2} \in(0, \infty)\right]$. Suppose that for some $\delta \in(1, \infty)$ and some $K<\infty$,

$$
f(h)-f^{\prime}(0) h \leqq K|h|^{1+1 / \delta}, \text { small }|h|,
$$

and suppose that with $2-\eta=\frac{\delta-1}{\delta+1} d$ and some $\varepsilon>0$,

$$
G(R) \equiv \sum_{|i| \leqq R} \operatorname{cov}\left(Y_{i}, Y_{j}\right) \geqq \varepsilon R^{2-\eta}, \quad \text { large } R .
$$

Then any limit in distribution $X$ of $\left\{\left(S_{n}-E\left(S_{n}\right)\right) / \sigma_{n}\right\}$, or equivalently of $\left\{\left(S_{n}-E\left(S_{n}\right)\right) / n^{d / 2}[G(n)]^{1 / 2}\right\}$, is non-Gaussian and satisfies, for some $c>0$ :

$$
\begin{aligned}
& \mathrm{E}(\exp (t X)) \leqq \exp \left(|c t|^{1+1 / \delta} /(1+1 / \delta)\right), \quad t \in \mathbb{R} ; \\
& \left.\begin{array}{l}
P(X \geqq x) \\
P(X \leqq-x)
\end{array}\right\} \leqq \exp \left(-(x / c)^{\delta+1} /(\delta+1)\right), \quad x \geqq 0 .
\end{aligned}
$$


Proof. This is an immediate consequence of Corollary 2.4 (with $N=1$ and $\theta_{1}=0$ ) together with (2.5), since (2.25) implies that $\left|f_{e}(h)\right| \leqq 2 K|h|^{1+1 / \delta}$.

When $d=d_{c}$, it is believed that $\delta$ and $\eta$ take on their classical values (as for $d$ $>d_{c}$ ) and thus that (2.24) is still an equality (as for $d<d_{c}$ ). The question of the nature of the scaling limit is complicated in this case by the possible existence of important logarithmic factors in the asymptotics of $f(h)$ and $G(R)$ [33]. The next two corollaries are intended to treat this problem in the Ising model context for an ordinary critical point in four dimensions and for a tricritical point in three dimensions. Both corollaries follow directly from Corollaries 2.3-2.4 (with $N=1$ ) together with (2.5).

Corollary 2.7. Assume the validity of (2.5) ( for some $c_{1}, c_{2}, K_{1}, K_{2} \in(0, \infty)$ ). Let $d$ $=4$, and suppose that for some $K, \varepsilon \in(0, \infty)$ and $\theta, \psi \in(-\infty, \infty)$,

$$
f(h)-f^{\prime}(0) h \leqq\left. K|h|^{4 / 3}|\log | h\right|^{\theta}, \quad \text { small }|h|,
$$

and

$$
G(R) \geqq \varepsilon R^{2}[\log R]^{\psi}, \quad \text { large } R ;
$$

then

$$
\psi \leqq \frac{3}{2} \theta \text {. }
$$

If $\psi=3 \theta / 2$, then any limit in distribution $X$ of $\left\{\left(S_{n}-E\left(S_{n}\right)\right) / \sigma_{n}\right\}$ is non-Gaussian and satisfies (1.11)-(1.13) for some $c>0$ with $p=4 / 3$ and $q=4$.

Corollary 2.8. Assume the validity of (2.5) ( for some $c_{1}, c_{2}, K_{1}, K_{2} \in(0, \infty)$ ). Let $d=3$, and suppose that for some $K, \varepsilon \in(0, \infty)$ and $\theta^{\prime}, \psi^{\prime} \in(-\infty, \infty)$,

$$
f(h)-f^{\prime}(0) h \leqq K|h|^{6 / 5}|\log | h||^{\theta^{\prime}}, \quad \text { small }|h|,
$$

and

$$
G(R) \geqq \varepsilon R^{2}[\log R]^{\psi^{\prime}}, \quad \text { large } R ;
$$

then

$$
\psi^{\prime} \leqq \frac{5}{3} \theta^{\prime}
$$

If $\psi^{\prime}=5 \theta^{\prime} / 3$, then any limit in distribution $X$ of $\left\{\left(S_{n}-E\left(S_{n}\right)\right) / \sigma_{n}\right\}$ is non-Gaussian and satisfies (1.11)-(1.13) for some $c>0$ with $p=6 / 5$ and $q=6$.

As was explained in the introduction to Sect. I, the above two corollaries can be regarded as giving sufficient conditions for rigorously proving that the scaling limit (at the critical dimension) is actually non-Gaussian. On the other hand, if one believes that the scaling limit is Gaussian (as is natural in the renormalization group approach) then these two corollaries can be regarded as simply giving certain critical point inequalities (2.31) and (2.34), which "must" be strict. Renormalization group calculations actually yield the value $\theta=1 / 3[24,29,37]$ and $\psi=0$, the latter based on the fact that the $\varepsilon$-expansion for $\eta$ has no terms of order $\varepsilon$ [26]. The calculations similarly suggest that $\psi^{\prime}=0$ while [30] $\theta^{\prime} \neq 0$. It should perhaps be pointed out though that the renormalization group calculations in some sense implicitly assume a priori that the scaling limit is Gaussian, since 
they are based on bifurcation expansions about the Gaussian fixed point. Earlier calculations [33] yield the relations, $\psi=(3 \theta-1) / 4$ and $\psi^{\prime}=\left(5 \theta^{\prime}-1\right) / 6$, which also imply that (2.31) and (2.34) are strict inequalities.

The next class of models we consider are those satisfying the GKS correlation inequalities $[13,23]$. Suppose $\left\{W_{j}: j \in \mathbb{Z}^{d}\right\}$ is obtained as a limit of finite systems $\left\{W_{1}^{m}, \ldots, W_{m}^{m}\right\}$ whose joint distribution on $\mathbb{R}^{m}$ is proportional to

$$
\exp \left(\sum_{i=1}^{\infty} \sum_{j_{1}, \ldots, j_{i}=1}^{m} J_{j_{1}, \ldots, j_{i}}^{m} w_{j_{1}} \ldots w_{j_{i}}\right) \prod_{k=1}^{m} d \varrho_{k}\left(w_{k}\right)
$$

where $J_{j_{1}, \ldots, j_{i}}^{m} \geqq 0$, and each $\varrho_{k}$ is an even measure on $\mathbb{R}^{1}$; then $\left\{W_{j}\right\}$ will satisfy the $G K S$ inequalities; i.e., for any $N, M, j_{1}, \ldots, j_{N}, k_{1}, \ldots, k_{M}$,

$\operatorname{cov}\left(W_{j_{1}} \ldots W_{j_{N}}, W_{k_{1}} \ldots W_{k_{M}}\right) \geqq 0$.

Suppose $Y_{k}=\sum_{i} \sum_{j_{1}, \ldots, j_{i}} \tilde{J}_{j_{1}, \ldots, j_{i}}^{k} W_{j_{1}} \ldots W_{j_{l}}$, with $\tilde{J}_{j_{1}, \ldots, j_{i}}^{k} \geqq 0$, and $S_{n}$ is defined by (2.1); then it easily follows from (2.36) that $\operatorname{cov}\left(Y_{j_{1}} \ldots Y_{j_{N}}, Y_{k_{1}} \ldots Y_{k_{M}}\right) \geqq 0$ and thus for $T=\sum Y_{i},(2.21)$ is valid for $z \geqq 0$. By the proof of Prop. 2.5, Hypothesis I will be valid for $z \in[0, \varepsilon]$ under the additional assumptions of translation invariance and the validity of Hypothesis A.

There are two situations in which Hypothesis I can be shown to be valid also for $z<0$. The first situation occurs when $J_{j_{1}, \ldots, j_{2}}=0$ for $i$ odd and $\tilde{J}_{j_{1}, \ldots, j_{i}}^{k}=0$ for $i$ even; here each $Y_{k}$ and $S_{n}$ has an even distribution so that $f_{n}$ and $f$ are even functions of $z$. In a typical such situation one would have $Y_{k}=W_{k}$; this yields a generalization of the FKG result of (2.18) and Proposition 2.5 to the case of multi-spin interactions (although with $h_{k} \equiv 0$ and each $\varrho_{k}$ even in (2.18)). The second situation occurs when for some $\varepsilon>0$, the random variables $\left\{W_{j}^{\left\{z_{k}\right\}}\right\}$ with joint distribution propositional to $\exp \left(\sum z_{k} Y_{k}\right) d \mu_{0}$ satisfies the GKS inequalities providing $z_{k} \geqq-\varepsilon \forall k$; here $d \mu_{0}$ is the joint distribution of $\left\{W_{j}\right\}$. To see that this suffices to obtain (2.21) for $z \in[-\varepsilon, 0$ ), we note that letting

$$
Y_{k}^{\left\{z_{k}\right\}}=\sum_{i} \sum_{j_{1}, \ldots, j_{i}} \tilde{J}_{j_{1}, \ldots, j_{i}}^{k} W_{j_{1}}^{\left\{z_{k}\right\}} \ldots W_{j_{1}}^{\left.z_{k}\right\}}
$$

we have for $z_{k}, z_{k}^{\prime} \leqq 0, z_{k}+z_{k}^{\prime} \geqq-\varepsilon$, that

$$
\begin{aligned}
\operatorname{cov}\left(\exp \left[\sum z_{k} Y_{k}\right], \exp \left[\sum z_{k}^{\prime} Y_{k}\right]\right) \\
=\left\{E\left(\exp \left[\sum\left(z_{k}+z_{k}^{\prime}\right) Y_{k}\right]\right)\right\}^{2} \\
\quad \cdot \operatorname{cov}\left(\exp \left[\sum-z_{k}^{\prime} Y_{k}^{\left\{z_{k}+z_{k}^{\prime}\right\}}\right], \exp \left[\sum-z_{k} Y_{k}^{\left\{z_{k}+z_{k}^{\prime}\right\}}\right]\right) \geqq 0 .
\end{aligned}
$$

One can now easily obtain (2.21) as desired. This second situation occurs in particular when $S_{n}$ is the energy contained in the block $\left\{k: 1 \leqq k_{i} \leqq d\right\}$ where one may take $\varepsilon$ to be the inverse temperature $\beta$; for example, in the nearest neighbor

Ising model in zero external field, one would take $Y_{k}=J \sum_{j} W_{k} W_{j}$ where the sum is over the nearest neighbors of $k$. We state the next corollary in the notation appropriate to this context; its proof is essentially identical to the proof of Corollary 2.2. Recall that we automatically assume Hypotheses $A, B, C, F$, and $I$. We also replace $z$ by $t$, where this variable is proportional to $T_{c}-T$ in this context. 
Corollary 2.9. Assume the validity of (2.5) (for some $c_{1}, c_{2}, K_{1}, K_{2} \in(0, \infty)$ ). Define $\alpha, \alpha^{\prime}$, and $\eta_{E}$ by

$$
\begin{aligned}
& 2-\alpha=\liminf _{t \rightarrow 0-}\left(\log \left(f(t)-f^{\prime}(0) t\right) / \log |t|\right), \\
& 2-\alpha^{\prime}=\liminf _{t \rightarrow 0+}\left(\log \left(f(t)-f^{\prime}(0) t\right) / \log t\right), \\
& 2-\eta_{E}=\limsup _{R \rightarrow \infty}(\log G(R) / \log R),
\end{aligned}
$$

then $\bar{\alpha} \equiv \max \left(\alpha, \alpha^{\prime}\right) \leqq 1$ and

$$
2-\eta_{E} \leqq \frac{d \bar{\alpha}}{2-\bar{\alpha}} \text {. }
$$

Remark. In [9] Fisher derives the inequality, $2-\eta_{E} \leqq d \alpha^{\prime}$, but notes that the stronger inequality, $2-\eta_{E} \leqq d \alpha^{\prime} /\left(2-\alpha^{\prime}\right)$, should be valid. When $\alpha \leqq \alpha^{\prime},(2.41)$ gives this stronger result. Theorem 1.3 states that when (2.41) is an equality (in the refined sense), then $\alpha=\alpha^{\prime}$. The next corollary gives one version of this fact along with some other results.

Corollary 2.10. Assume the validity of (2.5) and (2.6). Suppose for some $\bar{\alpha}, \kappa$, and $K<\infty$,

$$
f_{e}(t) \leqq K|t|^{2-\bar{\alpha}}|\log | t||^{\kappa}, \quad \text { small } t
$$

then for some $K^{\prime}<\infty$

$$
G(R) \leqq K^{\prime} R^{d \bar{\alpha} /(2-\bar{\alpha})}(\log R)^{2 \kappa /(2-\bar{\alpha})}, \quad \text { large } R .
$$

If for some $\varepsilon>0$,

$$
G(R) \geqq \varepsilon R^{d \bar{\alpha} /(2-\bar{\alpha})}(\log R)^{2 \kappa /(2-\bar{\alpha})}, \quad \text { large } R,
$$

then $\bar{\alpha} \in[0,1]$,

$$
0<\liminf _{t \rightarrow 0+} \frac{f(t)-f^{\prime}(0) t}{f(-t)+f^{\prime}(0) t} \leqq \limsup _{t \rightarrow 0+} \frac{f(t)-f^{\prime}(0) t}{f(-t)+f^{\prime}(0) t}<\infty
$$

so that $\alpha=\alpha^{\prime}=\bar{\alpha}$ (e.g., as defined by (2.38)-(2.39)), and any limit in distribution $X$ of $\left\{\left(S_{n}-E\left(S_{n}\right)\right) / \sigma_{n}\right\}$ satisfies (1.11)-(1.13) for some $c>0$ with $p=2-\bar{\alpha}$.

Proof. This corollary follows directly from Theorem 1.3 and Corollary 2.4 (with $N=1$ ),

Remark. In the standard two-dimensional Ising model at its critical point, the exact formula of Onsager for the free energy $f(t)$ (see, e.g., [34]) yields the fact that (2.42) is valid with $\bar{\alpha}=0$ and $\kappa=1$; thus $G(R)$ is $O(\log R)$ according to $(2.43)$. $G(R) / \log R$ can in fact be bounded away from 0 as $R \rightarrow \infty$ by using the calculations of [19]; it follows that the asymptotic distribution of the energy fluctuations in a cube of volume $n^{2}$ (scaled by $\sigma_{n} \sim \sqrt{n^{2} \log n}$ ) has a "Gaussian tail": $P(X \leqq x)$ $=O\left(\exp \left(-(x / c)^{2} / 2\right)\right)$. This does not necessarily imply that $X$ has a Gaussian distribution. 
The third class of models we consider are those satisfying reflection positivity $[11,20]$. Reflection positivity is closely related to Osterwalder-Schrader positivity [27] and the existence of a transfer matrix. It is valid for a large class of classical models (and some quantum ones) including ones with multi-component spins [11]. We shall say that $\left\{Y_{i}: i \in \mathbb{Z}^{d}\right\}$ is reflection positive if for each $k=1, \ldots, d$, any $n=1,2, \ldots$, any choice of $i^{1}, \ldots, i^{n} \in \mathbb{Z}^{d}$ with $\left(i^{j}\right)_{k} \geqq 1$ for all $j$, any real function $F$ on $\mathbb{R}^{n}$ such that $E\left(\left[F\left(Y_{i^{1}}, \ldots, Y_{i^{n}}\right)\right]^{2}\right)<\infty$,

$$
E\left(F\left(Y_{\Theta_{k^{i}}}, \ldots, Y_{\Theta_{k^{i}}}\right) F\left(Y_{i^{1}}, \ldots, Y_{i^{n}}\right)\right) \geqq 0,
$$

where $\Theta_{k}: \mathbb{Z}^{d} \rightarrow \mathbb{Z}^{d}$ is reflection about the plane $i_{k}=1 / 2$ as defined by $(2.20)$.

Proposition 2.11. Suppose $\left\{Y_{i}: i \in \mathbb{Z}^{d}\right\}$ is reflection positive, translation invariant (as in Proposition 2.5) and satisfies Hypothesis A. Then Hypothesis I is valid.

Proof. Reflection positivity and the Schwarz inequality together imply that if $T=\sum_{i} Y_{i}$, with the (finite) sum only over $i$ 's with $i_{k} \geqq 1$, then letting $\Theta_{k} T=\sum_{i} Y_{\Theta_{k} i}$, one has

$$
E\left(e^{z T}\right) \leqq\left[E\left(e^{z\left(\Theta_{k} T+T\right)}\right)\right]^{1 / 2}, \quad z \in \mathbb{R} .
$$

Comparing (2.47) with (2.21), we see that the proof of Proposition 2.5 directly yields the desired result.

Remark. Euclidean field theories from a particularly interesting class of models to which Proposition 2.11 and our other results can be applied. For example, we may define for $i \in \mathbb{Z}^{2}$,

$$
Y_{i}=\int_{i_{1}}^{i_{1}+1} \int_{i_{2}}^{i_{2}+1}: P(\phi):(x, y) d x d y,
$$

where $\phi(x, y)$ is the generalized random field corresponding to an infinite volume $\lambda P(\phi)_{2}$ model. The validity of reflection positivity in this context is a well-known fact [32] and the existence of phase transitions has been established [18] so that a critical phase should exist at some particular value $\lambda_{c}$ of $\lambda$. In this context (and using the notation of [32]) $f(z)=\alpha_{\infty}\left(\lambda_{c}-z\right)-\alpha_{\infty}\left(\lambda_{c}\right)$ so that (1.7) and (2.9) relate the behavior of $\alpha_{\infty}(\lambda)$ near $\lambda_{c}$ to the long-range correlation of the (interaction) energy density, $: P(\phi):(\mathbf{r}) \equiv H_{I}(\mathbf{r})$; for example, if

$$
\alpha_{\infty}(\lambda)-\alpha_{\infty}\left(\lambda_{c}\right)-\alpha_{\infty}^{\prime}\left(\lambda_{c}\right)\left(\lambda-\lambda_{c}\right) \sim\left|\lambda-\lambda_{c}\right|^{p}
$$

and

$$
\left\langle H_{I}(\mathbf{r}) H_{I}\left(\mathbf{r}^{\prime}\right)\right\rangle-\left\langle H_{I}(\mathbf{r})\right\rangle\left\langle H_{I}\left(\mathbf{r}^{\prime}\right)\right\rangle \sim\left|\mathbf{r}-\mathbf{r}^{\prime}\right|^{-\left(d-2+\eta_{I}\right)},
$$

then $2-\eta_{I} \leqq(2-p) d / p$ (where $d=2$ for the two space-time dimensional model).

We end this section by noting that since Hypothesis $I$ is valid for such a varied class of models, there is some hope that it may also be true for continuum models of fluids so that critical exponent inequalities could be rigorously proved for them. We also note that since reflection positivity is valid for certain quantum statistical mechanical models [11], it may very well be possible to modify the techniques presented here to obtain exponent inequalities for such quantum models. We similarly expect that there should be applications to various gauge field theories in which reflection positivity is valid $[5,1,17,28]$. 


\section{Proof of General Results}

We begin by giving three lemmas. In the first two lemmas, $W_{n}$ is a sequence of random variables and $\Phi_{n}(t)=E\left(\exp \left(t W_{n}\right)\right)$.

Lemma 3.1. Suppose for some $t_{1}, t_{2}>0, \Phi_{n}\left(-t_{1}\right)$ and $\Phi_{n}\left(t_{2}\right)$ are bounded uniformly in $n$; then $W_{n}$ is weakly sequentially compact. Moreover, if $W_{n_{k}} \stackrel{w}{\longrightarrow} W$, then $\Phi_{n_{k}}(z) \rightarrow E(\exp (z W))$ uniformly on compact subsets of $\left\{z:-t_{1}<\operatorname{Re} z<t_{2}\right\}$ and for any $j=1,2, \ldots, E\left(\left(W_{n_{k}}\right)^{j}\right) \rightarrow E\left(W^{j}\right)$.

Proof. $\Phi_{n}(t)$ is convex for real $t$ and $\left|\Phi_{n}(z)\right| \leqq \Phi_{n}(\operatorname{Re} z)$; thus

$$
\left|\Phi_{n}(z)\right| \leqq \max \left(\Phi_{n}\left(-t_{1}\right), \Phi_{n}\left(t_{2}\right)\right), \quad-t_{1} \leqq \operatorname{Re} z \leqq t_{2},
$$

so $\left|\Phi_{n}(z)\right|$ is uniformly bounded for $-t_{1} \leqq \operatorname{Re} z \leqq t_{2}$. Since $W_{n_{k}} \stackrel{w}{\longrightarrow} W$ if and only if $\Phi_{n_{k}}(i y) \rightarrow E(\exp (i y W))$ for all real $y$. the conclusions of the lemma follow by standard complex variable arguments.

Lemma 3.2. Suppose $\lim \sup \Phi_{n}(t) \leqq 1$ for $t=-t_{1}<0$ and for $t=t_{2}>0$; then $W_{n} \stackrel{w}{\longrightarrow} 0$.

Proof. By the compactness part of Lemma 3.1 it suffices to prove that if $W_{n_{k}} \rightarrow W$, then $\Phi(t) \equiv E(\exp (t W))=1$ for $t=i y$, all real $y$. By (3.1) and Lemma 3.1, we have $\Phi(t) \leqq 1$ for $t \in\left(-t_{1}, t_{2}\right)$. But $\Phi$ is convex and $\Phi(0)=1$; thus $\Phi(t)=1$ for $t \in\left(-t_{1}, t_{2}\right)$ and thus by analyticity $\Phi(i y)=1$ for all real $y$.

Lemma 3.3. If $X$ is a random variable such that for some $c>0$ and $p \in[1, \infty)$,

$$
E(\exp (t X)) \leqq \exp \left((c t)^{p} / p\right), \quad t \geqq 0 ;
$$

then

$$
P(X \geqq x) \leqq \exp \left(-(x / c)^{q} / q\right), \quad x \geqq 0,
$$

where $q \in(1, \infty]$ satisfies $(1 / q)+(1 / p)=1$. (For the case $p=1, q=\infty$, (3.3) is interpreted to mean $P(X>c)=0$.)

Proof. This is a standard result. It can easily be derived by using Chebyshev's inequality to obtain the estimate

$$
\begin{aligned}
P(X \geqq x) & =P\left(e^{t X} \geqq e^{t x}\right) \leqq e^{-t x} E\left(e^{t X}\right) \\
& \leqq \exp \left(-t x+(c t)^{p} / p\right), \quad t \geqq 0,
\end{aligned}
$$

and then minimizing the last expression over $t$.

Proof of Theorem 1.1. Let $W_{n}=S_{n} / V_{n}-f^{\prime}(0)$. By the convexity of $f_{n}$, it follows that $f_{n}(y) \leqq f_{n}^{\prime}(y)$. $y$ for all $y \in(-\varepsilon, \varepsilon)$ so that for all real $z$,

$$
\begin{aligned}
E\left(\exp \left(z W_{n}\right)\right) & =\exp \left(V_{n} f_{n}\left(z / V_{n}\right)-f^{\prime}(0) z\right) \\
& \leqq \exp \left(z\left[f_{n}^{\prime}\left(z / V_{n}\right)-f^{\prime}(0)\right]\right) .
\end{aligned}
$$

Since $f^{\prime}(0)$ exists and $V_{n} \rightarrow \infty$, and $f_{n} \rightarrow f$, standard convex function arguments show that $f_{n}^{\prime}\left(z / V_{n}\right) \rightarrow f^{\prime}(0)$ for all real $z$. Thus $\limsup _{n \rightarrow \infty} E\left(\exp \left(z W_{n}\right)\right) \leqq 1$ for all $z$ and consequently by Lemma $3.2, W_{n} \rightarrow 0$ as desired. 
Remark. The above proof can be easily modified to show that if Hypotheses $A$ and $D$ are valid and $E\left(S_{n}\right) / V_{n}=f_{+}^{\prime}(0) \equiv \lim _{h \rightarrow 0+}(f(h)-f(0)) / h$ for all $n$ then $S_{n} / V_{n} \longrightarrow f_{+}^{\prime}(0)$ even if $f^{\prime}(0)$ doesn't exist. A similar result holds with the right-hand derivative $f_{+}^{\prime}(0)$ replaced by the left hand derivative. "One-sided" extensions of Theorems 1.2 and 1.4 can also be made providing some additional hypotheses are assumed (such as that $\left.E\left(S_{n}-E\left(S_{n}\right)\right)^{4}=O\left(\sigma_{n}^{4}\right)\right)$.

Proof of Theorem 1.2. We let

$$
X_{n}=\left(S_{n}-E\left(S_{n}\right)\right) / \sigma_{n}=\left(S_{n}-V_{n} f_{n}^{\prime}(0)\right) / \sigma_{n}=\left(S_{n}-V_{n} f^{\prime}(0)\right) / \sigma_{n} .
$$

Now by Hypothesis $I, \Phi_{n}(t) \equiv E\left(\exp \left(t X_{n}\right)\right)$ satisfies

$$
\Phi_{n}(t)=\exp \left(V_{n}\left[f_{n}\left(t / \sigma_{n}\right)-f^{\prime}(0) t / \sigma_{n}\right]\right) \leqq \exp \left(V_{n} f_{+}\left(t / \sigma_{n}\right)\right) .
$$

If (1.7) were not valid, then since $f_{+}$is non-negative and $f_{e}(t)=f_{+}(t)+f_{+}(-t)$, we would have for some subsequence $n_{k}$ and some $t>0$ that $V_{n_{k}} f_{+}\left( \pm t / \sigma_{n_{k}}\right) \rightarrow 0$ so that by (3.7) and Lemmas 3.2 and 3.1, $E\left(\left(X_{n_{k}}\right)^{2}\right) \rightarrow 0$. But $E\left(\left(X_{n_{k}}\right)^{2}\right)=1$ for all $k$; thus (1.7) must be valid.

Remark. If one defines $\tilde{\sigma}_{n}=\left[E\left(S_{n}-E\left(S_{n}\right)\right)^{2 j}\right]^{1 / 2 j}$ with $j>1$ and $\tilde{X}_{n}=\left(S_{n}-E\left(S_{n}\right)\right) / \tilde{\sigma}_{n}$, then a slight variation of the above proof shows that (1.7) remains valid with $\sigma_{n}$ replaced by $\tilde{\sigma}_{n}$. This is (in principle) an improvement since $\tilde{\sigma}_{n} \geqq \sigma_{n}$.

Proof of Theorem 1.3. If (1.8) is valid, then $V_{n} f_{+}\left(+t_{0} / \sigma_{n}\right)$ and $V_{n} f_{+}\left(-t_{0} / \sigma_{n}\right)$ are uniformly bounded for some $t_{0}>0$ and thus by (3.7) and Lemma 3.1 any limit in distribution $X$ of $X_{n}$ has $E(\exp (t X)) \neq 1$ analytic in the strip $\operatorname{Re} t<t_{0}$. On the other hand, if (1.9) is not valid, then by (3.7) and the monotonicity of $f_{+}$on $(0, \varepsilon)$ and on $(-\varepsilon, 0)$ we would have for some limit $\tilde{X}$ of $X_{n}$ that $E(\exp (t \tilde{X}))=1$ either on $[0, s]$ or on $[-s, 0]$ for some $s>0$; this contradiction shows that (1.9) must be valid. We now assume (1.8) and Hypothesis $\mathrm{G}$ and note that by the monotonicity of $f_{ \pm}$, we have for some fixed $t>0$ and any $s>0$, constants $K, \delta \in(0, \infty)$ such that for all $n$, all $z \in[-t,-s] \cup[s, t]$,

$$
\delta \leqq V_{n} f_{ \pm}\left(z / \sigma_{n}\right) \leqq K,
$$

so that

$$
\delta / K \leqq f_{+}\left(z / \sigma_{n}\right) / f_{-}\left(z / \sigma_{n}\right) \leqq K / \delta .
$$

To obtain (1.10) from (3.9), it suffices to show that for some $r>0,\left\{\left[s / \sigma_{n}, t / \sigma_{n}\right]\right\}$ covers $(0, r)$. But this will be the case if for all sufficiently large $n, s / \sigma_{n} \leqq t / \sigma_{n+1}$. This inequality will be valid if we choose $s<t / \lim \sup \left(\sigma_{n+1} / \sigma_{n}\right)$, which completes the proof of Theorem 1.3.

Remark. A slight modification of the above proof shows that (1.10) remains valid if in Hypothesis $G$, we only assume that for some subsequence $n_{k}$, $\lim \sup _{k} \sigma_{n_{k+1}} / \sigma_{n_{k}}<\infty$.

Proof of Theorem 1.4. Let $X$ be any limit in distribution of $X_{n}$. By (3.7), Lemma 3.1, and the fact that $0 \leqq f_{+} \leqq f_{e}$, we have that

$E(\exp (t X)) \leqq \exp \left[\lim \sup V_{n} f_{e}\left(t / \sigma_{n}\right)\right], \quad$ all real $t$, 
if the right hand side is finite for all real $t$. Now by (1.8),

$$
\limsup _{n \rightarrow \infty} V_{n} f_{e}\left( \pm t_{0} / \sigma_{n}\right)<\infty
$$

for some $t_{0}>0$ and so by (1.3) there is a finite $c$ so that for any $t \neq 0$ we have

$$
\begin{aligned}
\limsup _{n \rightarrow \infty} V_{n} f_{e}\left(t / \sigma_{n}\right) & =\left(\frac{|t|}{t_{0}}\right)^{p} \limsup _{n \rightarrow \infty}\left[V_{n} f_{e}\left(t_{0} / \sigma_{n}\right) \cdot \frac{f_{e}\left(\frac{|t|}{t_{0}} t_{0} / \sigma_{n}\right)}{\left(\frac{|t|}{t_{0}}\right)^{p} f_{e}\left(t_{0} / \sigma_{n}\right)}\right] \\
& \leqq\left(\frac{|t|)^{p}}{t_{0}}\right)^{p} \limsup _{n \rightarrow \infty}\left[V_{n} f_{e}\left(t_{0} / \sigma_{n}\right)\right] \limsup _{s \rightarrow 0} \frac{f_{e}\left(\frac{|t|}{t_{0}} s\right)}{\left(\frac{|t|}{t_{0}}\right)^{p} f_{e}(s)} \\
& \leqq \frac{|t|^{p}}{\left|t_{0}\right|^{p}} \limsup _{n \rightarrow \infty}\left[V_{n} f_{e}\left(t_{0} / \sigma_{n}\right)\right] \cdot \sup _{z>0}\left(\limsup _{s \rightarrow 0} \frac{f_{e}(s z)}{z^{p} f_{e}(s)}\right) \\
& \leqq|c t|^{p} / p .
\end{aligned}
$$

In the first inequality of (3.12) we have used Hypothesis $F$. Thus we have (1.12) and by Lemma 3.1, since $E\left(X_{n}\right)=0, E\left[\left(X_{n}\right)^{2}\right]=1$ for all $n$, we have (1.11), while (1.13) follows from Lemma 3.3 applied to $+X$ and to $-X$. To see that $p \leqq 2$, we note that since $E\left(X^{2}\right)=1, E(\exp (t X))=1+t^{2} / 2+o\left(t^{2}\right)$ as $t \rightarrow 0$ so that (1.12) would be impossible for $p>2$. To see that $p \geqq 1$, we first note that if $p<1$, then it would follow from (1.12) that $\log E(\exp (t X)) /|t| \rightarrow 0$ as $|t| \rightarrow \infty$. On the other hand, it is a standard fact that $\lim _{|t| \rightarrow \infty} \frac{1}{|t|} \log E(\exp (t X))=\|X\|_{\infty}$ and $\|X\|_{\infty}>0$ since $E\left(X^{2}\right) \neq 0$; thus $p \geqq 1$. The last sentence of the theorem is proved by making slight modifications of the above arguments.

Remark. If in Theorem 1.4 one makes the additional "balancing" assumption that

$$
\gamma \equiv \lim _{z \rightarrow 0+} f_{+}(z) / f_{e}(z)
$$

exists; then by (1.9), $\gamma \in(0,1)$ and a slight modification of the above proof yields an improved version of (1.12) (and consequently of (1.13)):

$$
E\left(e^{t X}\right) \leqq \begin{cases}\exp \left(\left|c_{+} t\right|^{p} / p\right), & t \geqq 0 \\ \exp \left(\left|c_{-} t\right|^{p} / p\right), & t \leqq 0\end{cases}
$$

where $\left(c_{ \pm}\right)^{p} / p=\liminf _{k} V_{n_{k}} f_{ \pm}\left(1 / \sigma_{n_{k}}\right)$ and $n_{k}$ is any subsequence such that $X_{n_{k}} \stackrel{w}{\longrightarrow} X$. We also note that if in (1.3), the supremum over $z>0$ is replaced by a lim sup as $z \rightarrow \infty$, then (1.12) (resp. (1.13)) remains valid for large $|t|$ (resp. for large $x$ ). 


\section{References}

1. deAngelis, G.F., deFalco, D., Guerra, F.: Note on the abelian Higgs-Kibble model on a lattice: absence of spontaneous magnetization. Phys. Rev. D 17, 1624-1628 (1978)

2. Baker, G.A.,Jr.: Selfinteracting boson quantum field theory and the thermodynamic limit in $d$ dimensions. J. Math. Phys. 16, 1324-1346 (1975)

3. Baker, G.A.,Jr.: Renormalization group structure for translationally invariant ferromagnets. J. Math. Phys. 18, 590-607 (1977)

4. Blume, M., Emery, V.J., Griffiths, R.B.: Ising model for the $\lambda$ transition and phase separation in $\mathrm{He}^{3}-\mathrm{He}^{4}$ mixtures. Phys. Rev. A 4, 1071-1077 (1971)

5. Brydges, D., Fröhlich, J., Seiler, E. : On the construction of quantized gauge fields, I. general results. Preprint I.H.E.S. (1978)

6. Buckingham, M.J., Gunton, J.D.: Correlations at the critical point of the Ising model. Phys. Rev. 178, 848-853 (1969)

7. Benguigui, L., Schulman, L.S.: Topological classification of phase transitions. Phys. Lett. 45A, 315-316 (1973)

8. Ellis, R.S., Newman, C.M. : Fluctuationes in Curre-Werss exemplis. In: Mathematical problems in theoretical physics. Dell'Antonio, G., Doplicher, S., Jona-Lasinio, G. (eds.). Berlin, Heidelberg, New York: Springer 1978

9. Fisher, M.E.: Rigorous inequalities for critical-point correlation exponents. Phys. Rev. 180, 594-600 (1969)

10. Fisher, M.E.: General scaling theory for critical points. In: Collective properties of physical systems. Lundqvist, B., Lundqvist, S. (eds.). New York and London: Academic Press 1973

11. Fröhlich, J., Israel, R., Lieb, E.H., Simon, B. : Phase transitions and reflection positivity. I. General theory and long range lattice models. Commun. Math. Phys. 62, 1-34 (1978)

12. Fortuin, C., Kastelyn, P., Ginibre, J.: Correlation inequalities on some partially ordered sets. Commun. Math. Phys. 22, 89-103 (1971)

13. Griffiths, R.B.: Correlations in Ising ferromagnets. II. External magnetic fields. J. Math. Phys. 8, 484-489 (1967)

14. Gallavotti, G.: Probabilistic aspects of critical fluctuations. In : Critical phenomena. Brey, J., Jones, R.B. (eds.). Berlin, Heidelberg, New York: Springer 1976

15. Glimm, J., Jaffe, A. : Critical problems in quantum fields. In : Mathematical problems in theoretical physics. Dell'Antonio, G., Doplicher, S., Jona-Lasinio, G. (eds.). Berlin, Heidelberg, New York: Springer 1978

16. Glimm, J., Jaffe, A.: Critical exponents and elementary particles. Commun. Math. Phys. 52, 203-209 (1977)

17. Glimm, J., Jaffe, A.: Quark trapping for lattice $U(1)$ gauge fields. Phys. Lett. 66B, 67-69 (1977)

18. Glimm, J., Jaffe, A., Spencer, T.: Phase transitions for $\phi_{2}^{4}$ quantum fields. Commun. Math. Phys. 45, 203-216 (1975)

19. Hecht, R.: Correlation functions for the two-dimensional Ising model. Phys. Rev. 158, 557-561 (1967)

20. Hegerfeldt, G.C., Nappi, C.: Mixing properties in lattice systems. Commun. Math. Phys. 53, 1-7 (1977)

21. Jona-Lasinio, G.: Probabalistic approach to critical behavior. In: New developments in quantum field theory and statistical mechanics. Lévy, M., Mitter, P. (eds.). New York: Plenum 1977

22. Kadanoff, L.P.: Scaling laws for Ising models near $T_{c}$. Physics 2, 263-272 (1966)

23. Kelly, D.G., Sherman, S.: General Griffith's inequalities on correlations in Ising ferromagnets. J. Math. Phys. 9, 466-484 (1968)

24. Larkin, A.I., Khmel'nitskii, D.E.: Phase transition in uniaxial ferroelectrics. Sov. Phys. JETP 29, $1123-1128$ (1969)

25. Newman, C.M.: Short distance scaling and the maximal degree of a field theory. Phys. Lett. B (to appear)

26. Nelson, D.: Private communication

27. Osterwalder, K., Schrader, R.: Axioms for Euclidean Green's functions. Commun. math. Phys. 31, 83-112 (1973), Axioms for Euclidean Green's functions. II. Commun. Math. Phys. 42, 281-305 (1975)

28. Osterwalder, K., Seiler, E.: Gauge field theories on a lattice. Ann. Phys. 110, 440-471 (1978) 
29. Pfeuty, P., Toulouse, G.: Introduction to the renormalization group and to critical phenomena. New York: Wiley 1977 (Sect. 5.4)

30. Riedel, E.K.: Private communication

31. Schrader, R.: A possible constructive approach to $\phi_{4}^{4}$. Commun. math. Phys. 49, 131-153,(1976); A possible constructive approach to $\phi_{4}^{4}$ III. Commun. Math. Phys. 50, 97-102 (1976)

32. Simon, B.: The $P(\phi)_{2}$ Euclidean (quantum) field theory. Princeton: Princeton University Press 1974

33. Stell, G.: Extension of the Ornstein-Zernike theory of the critical region. Phys. Rev. Lett. 20, 533-536 (1968); Extension of the Ornstein-Zernike theory of the critical region. II. Phys. Rev. B 1, 2265-2270 (1970)

34. Thompson, C.: Mathematical statistical mechanics. New York: Macmillan 1972 (Sect. 5-5)

35. Wilson, K.G., Fisher, M.E.: Critical exponents in 3.99 dimensions. Phys. Rev. Lett. 28, 240-243 (1972)

36. Wilson, K.G., Kogut, J.: The renormalization group and the $\varepsilon$ expansion. Phys. Rep. 12, 75-200 (1975)

37. Wegner, F.J., Riedel, E.K.: Logarithmic corrections to the molecular-field behavior of critical and tricritical systems. Phys. Rev. B 7, 248-256 (1973)

Communicated by A. Jaffe

Received December 12, 1978 